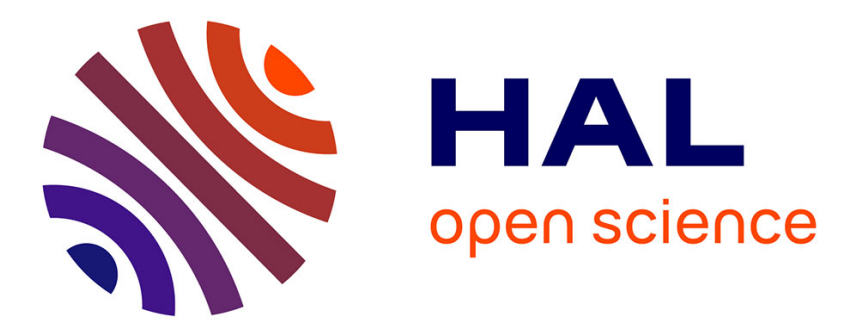

\title{
Luminescent latex particles loaded with anionic lanthanide complexes: a versatile platform for multicolour optical coding
}

N. Wartenberg, O. Raccurt, Daniel Imbert, M. Mazzanti, E. Bourgeat-Lami

\section{- To cite this version:}

N. Wartenberg, O. Raccurt, Daniel Imbert, M. Mazzanti, E. Bourgeat-Lami. Luminescent latex particles loaded with anionic lanthanide complexes: a versatile platform for multicolour optical coding. Journal of Materials Chemistry C, 2013, 1 (10), pp.2061. 10.1039/c3tc00756a . hal-01873155

\author{
HAL Id: hal-01873155 \\ https://hal.science/hal-01873155
}

Submitted on 4 Oct 2021

HAL is a multi-disciplinary open access archive for the deposit and dissemination of scientific research documents, whether they are published or not. The documents may come from teaching and research institutions in France or abroad, or from public or private research centers.
L'archive ouverte pluridisciplinaire HAL, est destinée au dépôt et à la diffusion de documents scientifiques de niveau recherche, publiés ou non, émanant des établissements d'enseignement et de recherche français ou étrangers, des laboratoires publics ou privés. 
Cite this: DOI: $10.1039 / \mathrm{c} 0 x \times 00000 x$

wWw.rsc.org/XXXXXX

ARTICLE TYPE

Luminescent latex particles loaded with anionic lanthanide complexes: versatile platforms for multicolour optical coding

\author{
N. Wartenberg ${ }^{a, b}$, O. Raccurt ${ }^{\text {b,c }}$, D. Imbert ${ }^{\mathrm{d}}$, M. Mazzanti ${ }^{\mathrm{d}}$, E. Bourgeat-Lamia ${ }^{\mathrm{a}}{ }^{*}$ \\ Received (in XXX, XXX) XthXXXXXXXXX 20XX, Accepted Xth XXXXXXXXX 20XX \\ ${ }_{5}$ DOI: 10.1039/b000000x
}

\begin{abstract}
Luminescent polymer latexes based on lanthanide complexes have been synthesized by miniemulsion polymerization. A self-assembled europium complex was embedded into poly(methyl methacrylate) nanoparticles without covalent linking and compared to a commercially-available neutral chelate. The nature of the surfactant was found to have a great impact on the incorporation process for the europium complex and only the latexes stabilized by 10 a cationic surfactant exhibited a luminescence signal. A maximum doping level of about $2 \%$ in weight in the final monodispersed particles was obtained. The resulting polymeric luminescent nanoparticles showed good stability over leakage. The described synthetic method was used to incorporate multiple lanthanide complexes into latexes nanoparticles affording multicolour nanolabels. Two series of polymeric latexes bearing codes are presented.
\end{abstract}




\section{INTRODUCTION}

The synthesis of luminescent nanoparticles is an attractive field that has received a lot of interest over the past decade [1-3]. A large variety of nanoparticles has already been developed and well described in the literature such as quantum dots [4], dye-doped silica [5] or polymeric nanoparticles [6]. The confinement of thousands of fluorescent molecules at the nanometer scale renders those kinds of particles particularly attractive for biological imaging due to their high sensitivity and selectivity [7]. Encapsulation of fluorophores into particles offers the possibility to functionalize the resulting particles, which is particularly useful for targeting applications $[8,9]$. More recently, the development of nanoparticles containing tags bearing codes has been extensively studied for multiplex bioassays $[10,11]$ or for products authentication and traceability to ensure the safety and quality of goods and to prevent counterfeits [12]. However, the capacity to read a lot of different emission simultaneously remains a major issue in this field. Indeed, the limitation for providing such complex codes based on luminescence is the number of luminescent molecules that one can effectively use before their emission signals overlap. One way to overcome this limitation is to use lanthanide chelates. Indeed, lanthanide complexes present numerous advantages over organic dyes such as large Stoke shifts, sharp emission spectra, long lifetimes and intrinsic protection against photobleaching [13]. The high potential of lanthanide complexes embedded into nanoparticles has been demonstrated for both silica [14, 15] and polymers [16]. Due to their versatile properties and applications, polymeric latexes are used in various areas including textiles, paints, coatings and bioassays [17, 18]. Different routes have been explored for adding fluorescent dyes to polymeric nanoparticles. Fluorescent dyes can be adsorbed onto the particle surface [19], copolymerized with the particles using luminescent monomers [20], introduced in the latex particles after their synthesis through diffusion $[21,22]$ or simply trapped inside the particles during polymerization [6]. It has been demonstrated that when luminescent molecules are covalently attached to the particles, they are less prone to leakage issues [2]. However, the chemical structure of the luminescent molecules has to be adapted to allow their covalent attachment to the polymer chains. This can sometime result, depending on the type of modification, on a change of the energy levels of the ligand resulting in a reduced efficiency of the lanthanide luminescence emission [13]. On the other hand, several studies have dealt with non-covalent incorporation by simple dissolution of the dye into the monomer followed by polymerization [11, 16]. This loading method is more flexible but then leakage issues may occur [21]. Due to the low water solubility of the luminescent dye, a very convenient process for non-covalent dye encapsulation is miniemulsion polymerization [23]. Miniemulsions are aqueous dispersions of kinetically stabilized submicronic (50-500 nm) monomer droplets prepared by using highshear devices such as ultrasounds or high-pressure homogenizers [24]. Miniemulsion droplets are stabilized against coalescence by using an appropriate surfactant (typically the same as in conventional emulsion polymerization), while monomer diffusion (e.g., Ostwald ripening) can be in principle retarded by the use of highly water-insoluble compounds (so-called hydrophobes) [25]. Hence, if the system is correctly formulated, the nanodroplets become the prevalent locus of nucleation [26]. This means that if one can directly dissolve some products like for instance lanthanide chelates in the monomer phase before it is dispersed, it should be possible to incorporate them into polymer particles through droplet nucleation without changing the identity of the droplets. Indeed, ideally, each of the monomer droplets is converted into a polymer particle of identical chemical composition. A large variety of compounds have been successfully incorporated into polymer latexes using this technique, but only few examples of lanthanide-containing polymer nanoparticles have been reported [11, 16, 27, 28]. Moreover these examples have been limited to neutral complexes. Polymeric nanoparticles incorporating lanthanide complexes capable of providing multiple, non-overlapping emission signals in different UV and Near-IR regions under a unique excitation wavelength are particularly desirable for the development of multimodal tags, bar-codes and lighting devices, but are rare. We have recently reported the synthesis of $\mathrm{Ln}(\text { pytz })_{3}\left(\mathrm{NHOct}_{3}\right)_{3}$ complexes based on a tridentate pyridine-tetrazolate ligand able to efficiently sensitize every luminescent lanthanide in the visible, the NIR or both regions [29, 30]. Here, we report the incorporation procedure for the anionic $\mathrm{Eu}^{\mathrm{III}}$ complex into poly(methyl methacrylate) (PMMA) nanoparticles through a miniemulsion polymerization process implemented for the incorporation of anionic complexes. We then demonstrate the possibility of the simultaneous incorporation of up to four lanthanide complexes into polymer particles leading to unique and complex optical signatures.

\section{EXPERIMENTAL SECTION}

\section{Materials}

Tris(dibenzoylmethane) $\quad \operatorname{mono}(1,10$-phenanthroline) europium(lll) $\left(\mathrm{Eu}(\mathrm{dbm})_{3}(\right.$ phen $\left.)\right)$, trioctylamine and $\mathrm{Ln}(\mathrm{III})$ trifluoromethanesulfonate $(\mathrm{Ln}=\mathrm{Eu}, \mathrm{Tb}, \mathrm{Sm}, \mathrm{Dy})$ were used as received from Aldrich. Methyl methacrylate (MMA), octadecyl acrylate (ODA), cetyl ammonium bromide (CTAB), sodium dodecyl sulfate (SDS), 2-2'-azobis(2methylpropionamidine) dihydrochloride (AIBA) and potassium persulfate (KPS) were all purchased from Acros Organics, and used without further purification. Lutensol AT-50 was received from BASF. Ultrapure water (18.2 $\mathrm{M} \Omega$ ) was used throughout the experiments. 


\title{
Cite this: DOI: 10.1039/c0xx00000x
}

\author{
www.rsc.org/xxxxxx
}

ARTICLE TYPE

\section{Preparation of $\operatorname{Ln}\left(\right.$ pytz $_{3}\left(\text { NHOct }_{3}\right)_{3}$}

The homoleptic trianionic lanthanide complexes $\mathrm{Ln}(\text { pytz })_{3}\left(\mathrm{NHOct}_{3}\right)_{3} \quad($ Scheme 1$)$ were prepared as previously described by reacting three equivalents of the 2,6-bis-tetrazolyl-pyridine ligand $\left(\mathrm{H}_{2}\right.$ pytz $)$ with one equivalent of lanthanide trifluoromethanesulfonate in methanol solution in the presence of trioctylamine and the solids were isolated according to the previously reported procedure [29].

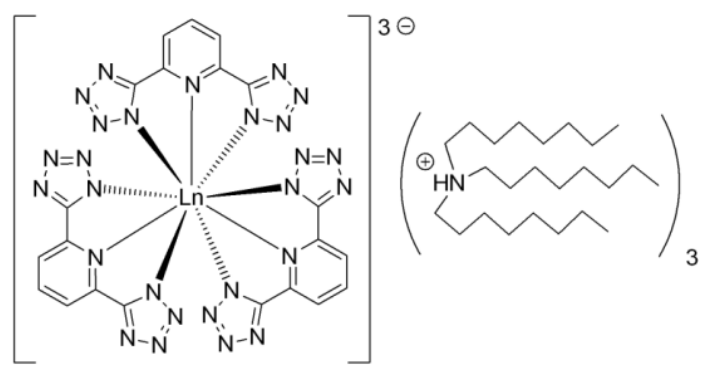

Scheme 1. Chemical structure of the homoleptic trianionic $\mathrm{Ln}(\text { pytz })_{3}\left(\mathrm{NHOct}_{3}\right)_{3}$ lanthanide complexes.

\section{Preparation of luminescent PMMA nanoparticles}

Two different lanthanide complexes were incorporated in polymeric nanoparticles. The neutral, commercially available $\mathrm{Eu}(\mathrm{dbm})_{3}$ (phen) lanthanide complex was used as reference and compared with the $\mathrm{Eu}(\text { pytz })_{3}\left(\mathrm{NHOct}_{3}\right)_{3}$ complex. Three kinds of surfactants were used to investigate their influence on the incorporation efficiency of both chelates. CTAB, SDS and Lutensol AT-50 were used as cationic, anionic and non-ionic surfactants, respectively. The amounts of the compounds used in the reactions are given in Table 1.

The lanthanide complexes were dissolved in a mixture of MMA and ODA. Then, the organic phase was added to the aqueous solution of surfactant under vigorous magnetic stirring. After 30 minutes of pre-emulsification, the solution was sonicated under magnetic stirring in an ice-cooled bath for 10 minutes at $80 \%$ amplitude (Branson sonifier 450, 750 W) to form miniemulsion droplets. Then, the miniemulsion was transferred in a three-necked $100 \mathrm{~mL}$ round-bottom flask equipped with a condenser. The reaction mixture was heated to $70^{\circ} \mathrm{C}$ under magnetic stirring and degassed with nitrogen for 15 minutes. The polymerization was initiated by KPS or AIBA (dissolved in $5 \mathrm{~g}$ of water and degassed) under nitrogen atmosphere. The reaction was allowed to continue between one and two hours depending on the surfactant used. A similar procedure was used to incorporate mixtures of lanthanide complexes into latex particles. In both cases, samples of final latexes were dialyzed against ultrapure water (3,5 kDa cut off, Roth) for 48 hours to remove unreacted materials and nonincorporated lanthanide complexes before analysis. Several aliquots from non-dialyzed solutions were collected after different periods of time for stability measurements. These aliquots were filtrated with Vivaspin $(5 \mathrm{kD}$ molecular weight cut-off) from Sartorius Biolab Products by centrifugation (15 minutes at $8500 \mathrm{rpm})$. The recovered solutions (free from particles) were analyzed by Inductively Coupled Plasma Mass Spectrometry (ICPMS, HP 4500 series) to evaluate the amount of lanthanide leaking out of the nanoparticles over time and the incorporation efficiency, which was defined as the percentage of lanthanide complex that was effectively incorporated in the particles compared to the quantity initially introduced.

Multicolour nanoparticles were prepared by mixing three and four isolated lanthanide complexes, Tb, Eu, Dy and Tb, $\mathrm{Eu}, \mathrm{Dy}, \mathrm{Sm}$, respectively. The chosen percentages of each metal ion used were calculated taking into account the values of the lanthanide complexes quantum yields 29.1, 70.2, 6.2 and 1.4 for Eu, Tb, Dy and Sm, respectively. The respective quantities used for the preparations of the colour codes with similar luminescence intensities for each lanthanide were: $1.8,4.7$ and $21.3 \mathrm{mg}$ for $\mathrm{Tb}, \mathrm{Eu}$ and $\mathrm{Dy}$ and: $0.4,1.1,5.0$ and $21.4 \mathrm{mg}$ for $\mathrm{Tb}, \mathrm{Eu}, \mathrm{Dy}$ and $\mathrm{Sm}$ corresponding to the ratios $1 / 3 / 12$ / for $\mathrm{Tb} / \mathrm{Eu} / \mathrm{Dy}$ and $1 / 3 / 12 / 56$ for $\mathrm{Tb} / \mathrm{Eu} / \mathrm{Dy} / \mathrm{Sm}$, respectively. The emission spectra of the two samples colour codes were recorded and normalized between 0 and 1 for comparison.

\section{Characterization}

Monomer conversion were determined gravimetrically by measuring the solids content of the final latexes. Dynamic Light Scattering (DLS, Nano-ZS from Malvern Instruments) was used to measure the droplet and particle sizes (intensity-averaged hydrodynamic diameters) and the polydispersity (indicated by the poly value - the higher this value, the broader the size distribution). In a typical experiment, samples were diluted in ultrapure water prior to measurement. The dialyzed latexes were characterized by Transmission Electron Microscopy (TEM) (Tecnai G2 STWIN, 200kV). Each latex sample was diluted and one drop of the solution was deposited on a carbon-coated copper grid. The sample was dried at ambient temperature and observed at an accelerating voltage of $200 \mathrm{kV}$. The luminescence spectra and lifetimes were recorded using a modular Fluorolog FL 3-22 spectrometer from HoribaJobin Yvon-Spex equipped with a double grating excitation monochromator and a iHR320 imaging spectrometer coupled to a R928P Hamamatsu photomultiplier. All 
spectra were corrected for detection and optical spectral response (instrumental functions) of the spectrofluorimeter.

Table 1.Standard procedure for the preparation of PMMA luminescent particles by miniemulsion polymerization.

\begin{tabular}{ll}
\hline Compound & Amount $(\mathrm{g})$ \\
\hline MMA & 10.0 \\
Lanthanide complex & 0.050 \\
ODA & 0.4 \\
SDS/Lutensol AT-50/CTAB & $0.075 / 0.15 / 0.075$ \\
KPS/AIBA $^{\mathrm{a}}$ & 0.1 \\
Water & 50 \\
\hline
\end{tabular}

a The nature of the initiator depends on the type of surfactant used. KPS was used for both SDS and Lutensol AT-50 while AIBA was used for CTAB

\section{RESULTS AND DISCUSSION}

\section{Preparation and characterization Eu(pytz)3(NHOct3)3-loadedPMMA latex particles}

of

The ionic $\mathrm{Eu}(\text { pytz })_{3}\left(\mathrm{NHOct}_{3}\right)_{3}$ chelate forms an ion-pair with the lipophilic trioctylamines and was therefore fully soluble in MMA. The resulting monomer solutions conserved their luminescent properties and were used to generate miniemulsion droplets in the presence of different kinds of surfactants. The results are summarized in Table 2 . All samples were colloidally stable and free of coagulum. The MMA conversion was limited to c.a. $90 \%$ in case of the non-ionic surfactant and close to $100 \%$ (within experimental error) for $\mathrm{CTAB}$ and SDS. The particle size was strongly dependent on the nature of the surfactant and was significantly larger for Lutensol AT-50 than for SDS or $\mathrm{CTAB}$ as expected. Indeed the stabilization of the miniemulsion by the non-ionic surfactant was less effective [31], leading to an important coalescence phenomenon during the polymerization. The limiting conversion observed for this particular experiment thus probably resides in the larger droplet/particle sizes. Obviously, the non-ionic surfactant does not satisfy the ideal scenario of a one-to-one copy of droplets into particles as attested by the low ratio of the number of particles to the number of droplets, $\left(\mathrm{N}_{\mathrm{p}} / \mathrm{N}_{\mathrm{d}}\right.$, Table 2). If there is a deviation from unity in this ratio, then it is preferable that it be slightly less than one rather than slightly greater than one. In the second scenario, if $\mathrm{N}_{\mathrm{p}} / \mathrm{N}_{\mathrm{d}}$ is greater than one, this implies that new particles have been created during the reaction and these new particles would not contain lanthanide chelates. If $\mathrm{N}_{\mathrm{p}} / \mathrm{N}_{\mathrm{d}}$ is lower than one, this means on the contrary that the miniemulsion suffered either droplet-droplet or dropletparticle coalescence which should guaranty that all final latex particles contain some lanthanide complex. In order to confirm the successful encapsulation of the lanthanide complex, the latexes were thoroughly dialyzed against ultrapure water and their emission and excitation spectra were recorded. Europium luminescence was only observed for PMMA nanoparticles stabilized by the cationic surfactant (Figure 1). Indeed, for both Lutensol AT-50 and SDS, the europium signal completely disappeared after dialysis. This result demonstrates that $\mathrm{Eu}(\text { pytz })_{3}\left(\mathrm{NHOct}_{3}\right)_{3}$ was not incorporated into the polymer latex particles when the latter were stabilized by anionic or non-ionic surfactants. In contrast, the polymer nanoparticles stabilized with $\mathrm{CTAB}$ exhibited a strong luminescence after dialysis as shown in Figure 1. Furthermore, it should be noted that the observed maximum emission at $617 \mathrm{~nm}$ and the shape of the emission and excitation spectra are in agreement with that of the europium chelate in methanol [29].

Time-resolved luminescence experiments were also carried out on the doped latex and $\mathrm{Eu}(\text { pytz })_{3}\left(\mathrm{NHOct}_{3}\right)_{3}$ dissolved in methanol to establish whether the fluorescence emission was affected by the PMMA environment. Intensity decay curves could be fitted to single exponentials, yielding luminescence lifetimes of $3.38 \mathrm{~ms}$ and $2.92 \mathrm{~ms}$ in methanol and PMMA, respectively (Figure S1, Supporting Information). The identical emission spectra (transitions and splitting analysis) and lifetimes found in solution and in the latex particles indicate that the embedded complexes remained intact.

In the literature, several neutral lanthanide complexes have been successfully incorporated into polymer latex using various types of surfactants without mention of any difficulty $[11,16,27]$. The solubility of the luminescent molecule into the monomer seemed to be sufficient to ensure efficient incorporation. The fact that both non-ionic and anionic surfactants lead to non-luminescent polymeric nanoparticles for $\mathrm{Eu}(\mathrm{pytz})_{3}\left(\mathrm{NHOct}_{3}\right)_{3}$ although the latter is fully soluble in MMA, is likely to reside in the anionic character of the complex. In order to confirm this assumption, two experiments were performed in exactly the same way as for the CTAB and SDS experiments, except that $\mathrm{Eu}(\mathrm{pytz})_{3}\left(\mathrm{NHOct}_{3}\right)_{3}$ was replaced by $\mathrm{Eu}(\mathrm{dbm})_{3}(\mathrm{phen})$, which is fully soluble in MMA and exhibits a non-ionic structure. In both cases, the final dialyzed latexes were found to be luminescent indicating successful dye incorporation regardless of the nature of the surfactant (Figure S2, Supporting Information).

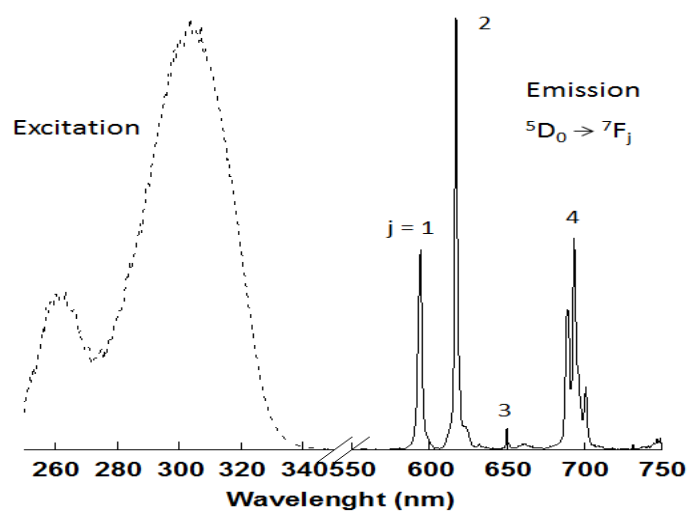

Figure 1. Excitation and emission spectra of Eu(pytz $)_{3}\left(\mathrm{NHOct}_{3}\right)_{3^{-}}$ loaded PMMA latex particles stabilized by CTAB $($ Kan $=617 \mathrm{~nm}$ and $\mathrm{\Lambda em}=300 \mathrm{~nm}$ ). 


\title{
Cite this: DOI: $10.1039 / \mathrm{c0xx00000x}$
}

\author{
www.rsc.org/xxxxxx
}

ARTICLE TYPE

\section{Elucidation of the role of the surfactant}

The above results show that we did not succeed in incorporating $\mathrm{Eu}(\mathrm{pytz})_{3}\left(\mathrm{NHOct}_{3}\right)_{3}$ into PMMA latex particles when SDS or Lutensol AT-50 were used as surfactants, even though the complex was fully soluble in MMA. We believe that the trioctylammonium cations that are responsible for the chelate solubility in MMA, interact with the water phase at the monomer droplet/water interface during droplet formation. This could lead to the exclusion of the resulting anionic complex from the monomer droplets. In order to confirm this assumption, we have performed partitioning experiments to estimate the fraction of europium that can migrate in the water phase prior to polymerization. Four mixtures (three containing surfactant and one without surfactant) were prepared according to the same protocol used before except that they were not polymerized. To allow phase separation, ODA was removed from the formulation and the mixtures containing surfactant were not sonified. After decantation, the quantity of Eu(III) into the water phase was determined by ICPMS. A reference experiment in pure water was also conducted in parallel to estimate the water solubility of $\mathrm{Eu}(\text { pytz })_{3}\left(\mathrm{NHOct}_{3}\right)_{3}$. The percentages of Eu(III) located in the water phase in the different cases are presented in Figure 2.

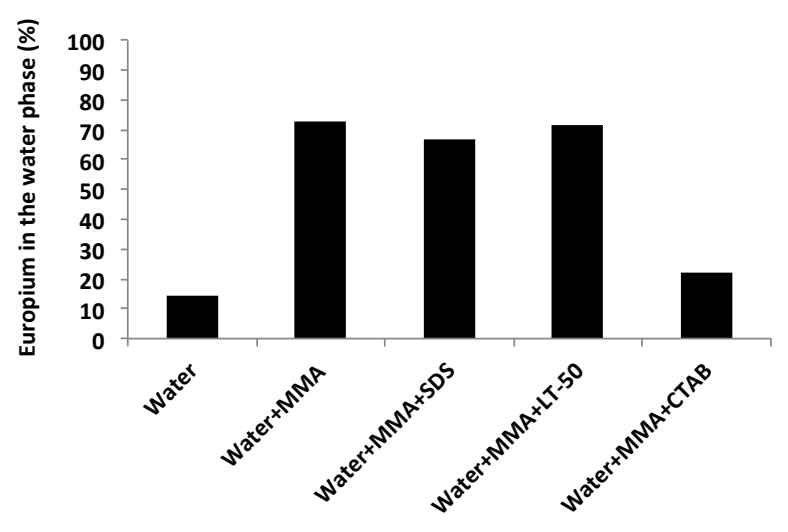

Figure 2. Estimation of Europium partitioning between water and MMA before polymerization for different formulations.

When SDS or Lutensol AT-50 were used as surfactants, an important quantity of $\mathrm{Eu}(\mathrm{III})$ was found to be localized into the water phase. The same behavior was observed in the absence of surfactant, indicating that SDS and Lutensol AT50 have no significant influence on $\mathrm{Eu}\left(\mathrm{pytz}_{3}\left(\mathrm{NHOct}_{3}\right)_{3}\right.$ partitioning. In contrast, the $\mathrm{Eu}(\mathrm{III})$ quantity into the water phase was found to be much lower when $\mathrm{CTAB}$ was used as surfactant. In this case, the $\mathrm{Eu}(\mathrm{III})$ concentration in the water phase was comparable to the $\mathrm{Eu}\left(\mathrm{pytz}_{3}\left(\mathrm{NHOct}_{3}\right)_{3}\right.$ water solubility and the slight difference between the two concentrations can be attributed to the MMA water solubility. These results indicate that CTAB could prevent the ionic lanthanide complex from diffusing out of the monomer phase during droplets formation by forming an electrostatic barrier at the monomer droplets/water interphase. In the event that the lanthanide complex could nevertheless migrate to the interface and dissociate, the resulting negatively charged europium chelate would instantaneously recombine with the CTAB molecules adsorbed at the surface of the droplets, which would restabilize the complex. In contrast, when SDS or Lutensol AT-50 were used as surfactants, the lanthanide complex could come in contact with water resulting in the loss of trioctylammonium ions and to a negatively charged lanthanide chelate, no more soluble in MMA. Consequently in those cases, lanthanide chelates are excluded from monomer droplets and the incorporation failed. It is worth noting here that $\mathrm{Eu}\left(\mathrm{pytz}_{3}\left(\mathrm{NHOct}_{3}\right)_{3}\right.$ is only partially soluble in water due to the hydrophobic character of the trioctylammonium cations. In contrast, when the lanthanide complex was first solubilized in MMA, the ionic europium chelate could better interact with water as the contact interfacial area was much higher, and thus dissociation of the trioctylammoniumcations was more important in this case.

Properties of Eu(pytz) $)_{3}\left(\mathrm{NHOct}_{3}\right)_{3}$-loaded PMMA latex particles stabilized by CTAB

Next, we examined the maximum loading capacity of the PMMA nanoparticles stabilized by CTAB, and their resulting luminescence properties. Several amounts of $\mathrm{Eu}(\text { pytz })_{3}\left(\mathrm{NHOct}_{3}\right)_{3}$ from 0.3 to $2 \mathrm{wt} \%$ (based on monomer) were introduced into MMA, and the resulting mixtures were sonified and polymerized. In all experiments, MMA conversion reached $100 \%$ after one hour. All miniemulsions were stable and exhibited a gradual increase in both initial droplet and final particle sizes with increasing the $\mathrm{Eu}(\mathrm{pytz})_{3}\left(\mathrm{NHOct}_{3}\right)_{3}$ content (e.g., $\mathrm{N}_{\mathrm{p}} / \mathrm{N}_{\mathrm{d}}$ remained almost constant) except, for the largest amount where the particles started to aggregate (Table 3). Indeed at $2 \mathrm{wt} \%$, the particle size strongly increased during the polymerization (e.g. $\mathrm{N}_{\mathrm{p}} / \mathrm{N}_{\mathrm{d}}$ significantly decreased) and the latex was found to be highly viscous and polydisperse, indicating that a destabilization occurred. Consequently, we did not attempt to increase further the amount of lanthanide complex, as we 
could not get stable particles for higher loadings. Ritcey et al. [16] found similar results when increasing the amount of europium complex above $2 \mathrm{wt} \%$ during miniemulsion polymerization of styrene stabilized by SDS. In the present study, the efficiency of the incorporation was found to be constant at $87.0 \pm 1.6 \%$ in the entire range of concentrations investigated indicating a very efficient incorporation whatever the amount of complex. In addition, TEM analysis (Figure 3) and DLS measurements showed that the particles were spherical and monodisperse (poly values lower than 0.1, Table 3). As seen in Figure 3a, no black spots could be observed at low loading contents indicating homogeneous incorporation of the lanthanide complex. In contrast, a few black spots could be identified inside the PMMA nanoparticles for higher loading contents (Figure 3b) probably due to slight aggregation of the europium chelates, which may facilitate their observation.

Table 3 Miniemulsion polymerization of MMA stabilized by CTAB in the presence of various amounts of Eu(pytz $)_{3}\left(\mathrm{NHOct}_{3}\right)_{3}$.

\begin{tabular}{|c|c|c|c|c|c|c|}
\hline $\begin{array}{c}\mathrm{Eu}(\mathrm{pytz})_{3}\left(\mathrm{NHOct}_{3}\right)_{3} \\
\text { content }(\mathrm{wt} \%)^{\mathrm{a}}\end{array}$ & $\begin{array}{c}\text { Monomer } \\
\text { conversion }(\%)\end{array}$ & $\begin{array}{c}\text { Droplet } \\
\text { diameter }(\mathrm{nm})^{\mathrm{b}}\end{array}$ & $\begin{array}{c}\text { Particle } \\
\text { diameter }(\mathrm{nm})^{\mathrm{b}}\end{array}$ & Poly value $^{\mathrm{b}}$ & $\mathrm{N}_{\mathrm{p}} / \mathrm{N}_{\mathrm{d}}$ & $\begin{array}{c}\text { Incorporation Efficiency } \\
(\%)\end{array}$ \\
\hline 0 & 97.7 & 89 & 105 & 0.028 & 0.66 & - \\
\hline 0.3 & 99.9 & 102 & 122 & 0.013 & 0.47 & 85.7 \\
\hline 0.5 & 99.7 & 119 & 136 & 0.050 & 0.54 & 87.5 \\
\hline 0.8 & 99.9 & 106 & 132 & 0.010 & 0.42 & 88.5 \\
\hline 1.0 & 99.9 & 140 & 144 & 0.015 & 0.74 & 87.6 \\
\hline 1.6 & 100 & 167 & 174 & 0.010 & 0.71 & 87.0 \\
\hline 2.0 & 99.8 & 223 & 376 & 0.437 & 0.17 & 85.4 \\
\hline
\end{tabular}

${ }^{a}$ Initial content relative to MMA. ${ }^{\mathrm{b}}$ Intensity-averaged hydrodynamic diameter and size dispersity determined by DLS
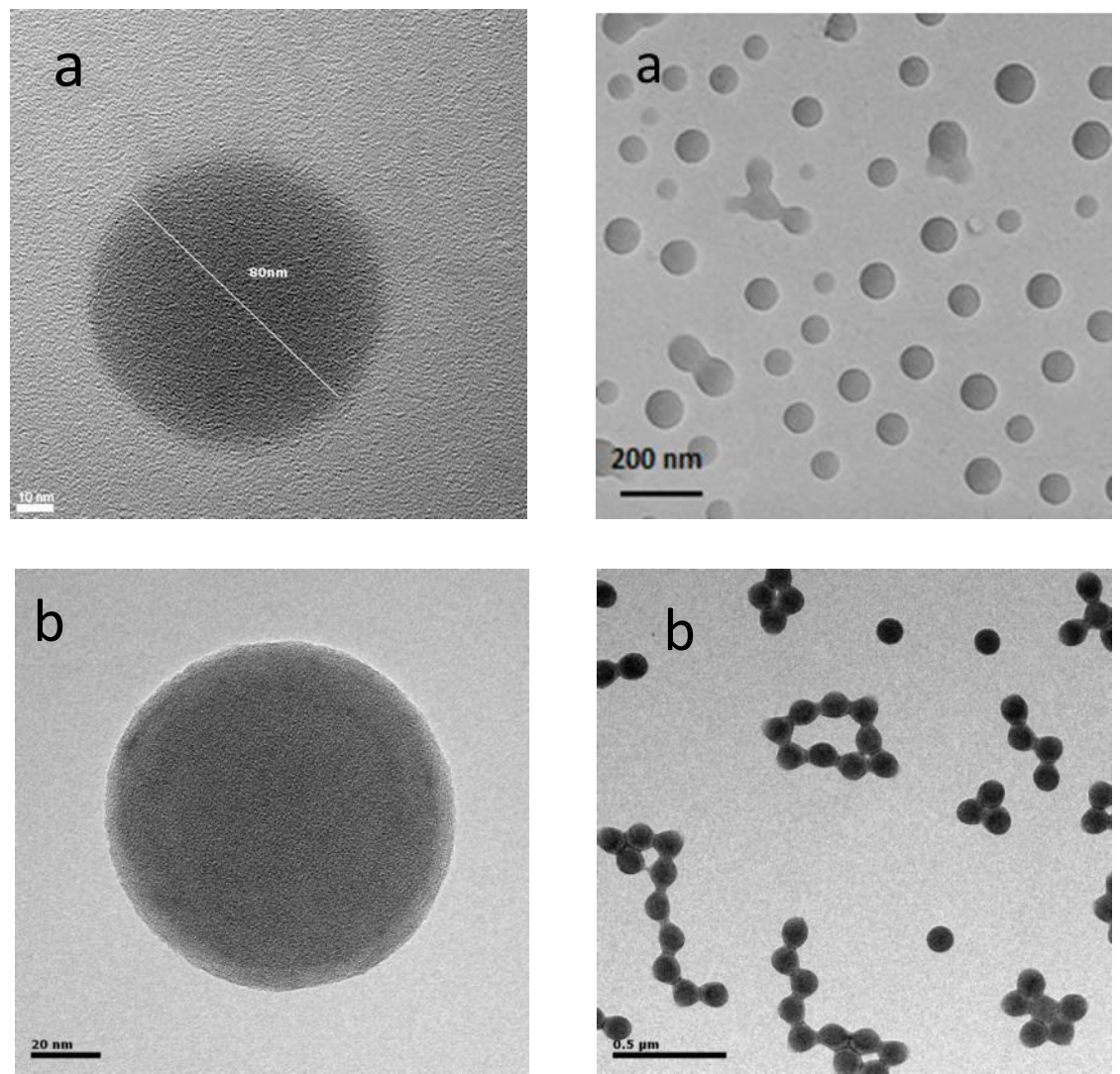

Figure 3. TEM images of PMMA latex particles doped with a) $0.3 \mathrm{wt} \%$ and b) $1.6 \mathrm{wt} \%$ of Eu(pytz) $)_{3}\left(\mathrm{NHOct}_{3}\right)_{3}$ 


\title{
Cite this: DOI: $10.1039 / \mathrm{c0xx00000x}$
}

\author{
www.rsc.org/xxxxxx
}

ARTICLE TYPE

Figure 4 shows the evolution of the luminescence intensity with increasing the amount of lanthanide chelates introduced. The intensity increased gradually with increasing the quantity of lanthanide complex. The corresponding lifetimes were found to 5 be constant at $2.88 \pm 0.11 \mathrm{~ms}$, which proves that no quenching occurred in the whole range of concentrations investigated. This behavior was confirmed by quantum yield measurements, which also remained constant (Table 4). The strong binding of the large bulky organic ligand prevents intermetallic energy transfers from 10 occurring. This is consistent with the high quantum yields measured for the pure complexes in the solid state [29]. Nevertheless absorption of the PMMA matrix was found to be quite high as indicated by the $20 \%$ decrease of the quantum yield. Based on the particle sizes and the solid contents, the 15 number of complexes per particle could be estimated. Each latex particle contained between c.a. $10^{3}$ and $10^{4}$ chelated lanthanide ions.

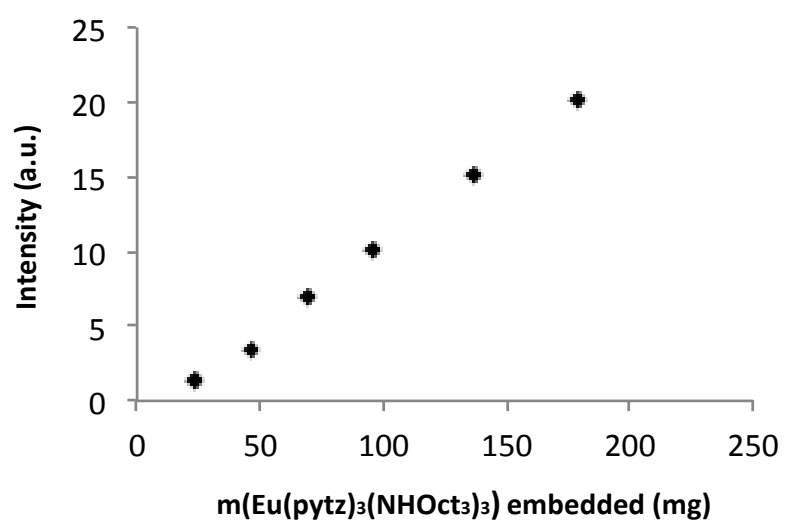

20 Figure 4. Luminescence intensity at $617 \mathrm{~nm}$ as a function of the $\mathrm{Eu}(\mathrm{pytz})_{3}\left(\mathrm{NHOct}_{3}\right)_{3}$ content of the latexes for a fixed solids content of $0.15 \%$

Table 4. Number of Eu(pytz) $)_{3}\left(\mathrm{NHOct}_{3}\right)_{3}$ complexes incorporated per ${ }_{25}$ particles and corresponding quantum yields.

\begin{tabular}{cccc}
\hline $\begin{array}{c}\text { Eu(pytz) })_{3}\left(\mathrm{NHOct}_{3}\right)_{3} \mathrm{co} \\
\text { ntent }(\mathrm{wt} \%)^{\mathrm{a}}\end{array}$ & $\begin{array}{c}\text { Quantum Yield }(\%) \\
\text { b }\end{array}$ & $\begin{array}{c}\text { Number of complexes } \\
\text { per particle }^{\mathrm{d}}\end{array}$ \\
\hline 0.3 & 39 & 60 & 841 \\
0.5 & 41 & 63 & 2329 \\
0.8 & 40 & 60 & 3115 \\
1.0 & 38 & 58 & 5567 \\
1.6 & 40 & 61 & 14321 \\
2.0 & 39 & 60 & $-{ }^{\mathrm{e}}$ \\
\hline
\end{tabular}

${ }^{a}$ Initial content relative to MMA. ${ }^{b}$ Measured with water as reference. ${ }^{c}$ Measured with PMMA as reference. ${ }^{\mathrm{d}}$ Calculated from DLS particle size and solids content. ${ }^{\mathrm{e}}$ Not calculated due to the high polydispersity.

30 In order to evaluate the stability over leakage of the lanthanide complex-loaded polymer particles, the europium concentration in the recovered serums (aliquots of water phase free from particles) was determined two weeks and two months after synthesis, and compared to the one after polymerization. The results of Figure 5 35 show that no leakage occurred in ultrapure water in ambient conditions within the experimental error. Indeed the europium concentration in the water phase was equal to $13.0 \pm 1.5 \%$ after two weeks and to $14.4 \pm 1.2 \%$ after two months whose values are very close to the one determined immediately after synthesis (e.g. $4013.0 \pm 1.6 \%)$. Then, it can be concluded that the incorporation was stable over at least two weeks.

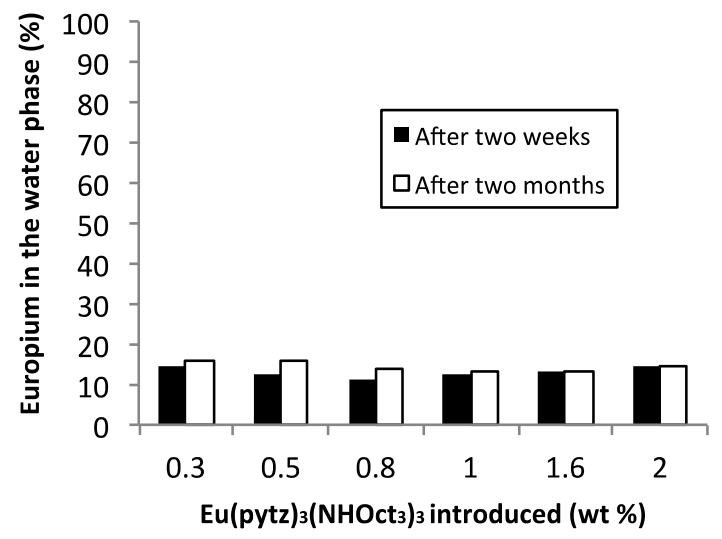

Figure 5. Europium concentrations in the serum (in percentage of the total 45 amount introduced) after two weeks and two months as a function of the initial content of $\mathrm{Eu}(\mathrm{pytz})_{3}\left(\mathrm{NHOct}_{3}\right)_{3}(\%$ by weight relative to MMA).

\section{Example of multicolor nanoparticle based on lanthanides emission}

${ }_{50} \mathrm{Eu}(\text { pytz })_{3}\left(\mathrm{NHOct}_{3}\right)_{3}$ was used to investigate the incorporation process. It is not expected that the change in lanthanide will affect the synthesis. Then, two mixtures of $\mathrm{Ln}(\text { pytz })_{3}\left(\mathrm{NHOct}_{3}\right)_{3}$ with $\mathrm{Ln}$ $=\mathrm{Tb}, \mathrm{Eu}, \mathrm{Dy}$ and $\mathrm{Ln}=\mathrm{Tb}, \mathrm{Eu}, \mathrm{Dy}, \mathrm{Sm}$, respectively, were incorporated into PMMA particles. The same protocol used 55 before was followed and after dialysis, luminescent properties of the resulting latexes were measured. As it is shown in Figure 6, characteristic emission of each lanthanide can be distinguished. The two samples showed a well-resolved fluorescence with not measurable intermetallic quenching arising from the mixing of 60 the different lanthanide complexes. This result demonstrates that the present system can be used to tailor the emission properties of latex nanoparticles under a single excitation wavelength. 


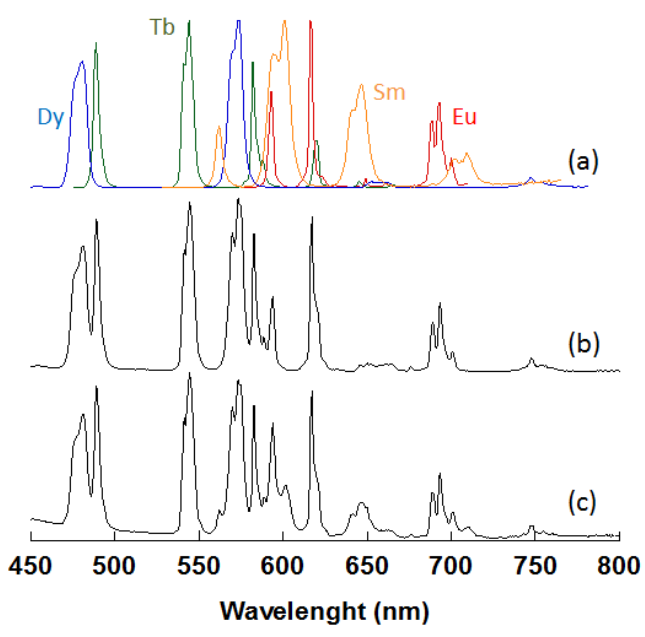

Figure 6. Normalized emission spectra of a) $\mathrm{Ln}\left(\right.$ pytz $_{3}\left(\mathrm{NHOct}_{3}\right)_{3}$ in methanol $\left(2.3 \cdot 10^{-3} \mathrm{M}, \mathrm{Ln}=\mathrm{Sm}, \mathrm{Eu}, \mathrm{Tb}, \mathrm{Dy}\right)$ and b, c) $\mathrm{Ln}(\text { pytz })_{3}\left(\mathrm{NHOct}_{3}\right)_{3}$-loadedPMMA latex particles under $300 \mathrm{~nm}$ irradiation.Ln=Eu,Tb,Dy (b) and $\mathrm{Ln}=\mathrm{Eu}, \mathrm{Tb}, \mathrm{Dy}, \mathrm{Sm}(\mathrm{c})$.

\section{CONCLUSIONS}

We described the non-covalent incorporation into PMMA latex particles of a series of original $\mathrm{Ln}^{\mathrm{III}}$ ion-pair complexes based on a ligand able to sensitize efficiently over ten lanthanide ions after 10 excitation under the same wavelength. This particular chelate was compared to a commercially available neutral complex similar to the ones commonly reported in the literature. It was found more challenging to incorporate the anionic than the neutral lanthanide chelates although both complexes were fully soluble in MMA. 15 Indeed, while the neutral lanthanide complex could be successfully introduced into PMMA nanoparticles regardless of the nature of the surfactant, the Eu(pytz $)_{3}\left(\mathrm{NHOct}_{3}\right)_{3}$ chelate was not totally immune to its immediate environment, and required an appropriate choice of surfactant to maintain it inside the particles.

20 Hence, a successful incorporation without covalent attachment and with up to $87 \%$ efficiency was achieved in this case when using a cationic surfactant that provided an electrostatic barrier preventing the chelate from diffusing in the water phase. This provides an unprecedented route to the incorporation of ionic 25 lanthanide complexes into polymer nanoparticles. Since many highly luminescent complexes reported in the literature are ionic [31-35], this opens a wide range of possibility for the design of new luminescent nanoparticles. The europium-based latex nanoparticles show bright luminescence with high quantum 30 yields independently of the amount of lanthanide complex introduced (in the range $0-2 \mathrm{wt} \%$ based on polymer). The resulting encoded latex suspensions were found to contain a significant number of lanthanide ions uniformly distributed within the particles. The latexes also proved to have excellent 35 colloidal stability similar to that of the bare latex suspension and good stability over leakage. The same procedure can be used to incorporate a series of lanthanide ions emitting at different wavelengths affording multicolour latex labels. As a proof of concept, multiple different emission spectra could be obtained, 40 with the same excitation wavelength, by mixing up to four lanthanides and by adjusting their molar ratio. Indeed contrary to organic dyes, which possess large emission spectra, sharp lanthanide emissions allow their simultaneous use without overlapping issues. As it is theoretically possible to produce 45 millions of codes by varying the amount of lanthanide ions, the emission range as well as the intensity and time-scale levels, this platform is particularly promising for multiplex detection, and could find applications in biomedicine, in materials coding or in optical photonic devices.

\section{${ }_{50}$ Notes and references}

${ }^{a}$ Université de Lyon, Univ. Lyon 1, CPE Lyon, CNRS UMR5265, Laboratoire de Chimie, Catalyse, Polymères et Procédés (C2P2), LCPP group, 43 Bd. du 11 Novembre 1918, F-69616, Villeurbanne, France.Fax: 33 472431768; Tel: 33 472431777; E-mail:

55 bourgeat@lcpp.cpe.fr

${ }^{b}$ Laboratoire de Chimie et de Sécurité des Nano Matériaux, DTNM/LITEN/CEA Grenoble, 17 rue des Martyrs, 38054 Grenoble cedex 9, France. E-mail: wartenbergnicolas@ hotmail.fr

${ }^{c}$ Current address: CEA LITEN, National Institute of Solar Energy INES, 60 Solar Technologies Department, Thermal Systems Laboratory, 73377 Le Bourget-du-Lac, France.E-mail: olivier.raccurt@cea.fr

${ }^{d}$ Laboratoire de Reconnaissance Ionique et Chimie de Coordination, Service de Chimie Inorganique et Biologique (UMR E-3 CEA/UJF), CEA-Grenoble, INAC, 17 rue des Martyrs, 38054 Grenoble cedex 9, 65 France.daniel.imbert@cea.fr; marinella.mazzanti@cea.fr

$\dagger$ Electronic Supplementary Information (ESI) available: [lifetime of $\mathrm{Eu}(\text { pytz })_{3}\left(\mathrm{NHOct}_{3}\right)_{3}$-loaded PMMA latex particles, excitation and emission spectra of $\mathrm{Eu}(\mathrm{dbm})_{3}($ phen)-loaded PMMA latex particles 70 stabilized by SDS, emission spectra of $\mathrm{Ln}(\text { pytz })_{3}\left(\mathrm{NHOct}_{3}\right)_{3}$ in methanol $(\mathrm{Ln}=\mathrm{Sm}, \mathrm{Tb}, \mathrm{Eu}$ and $\mathrm{Dy})$ and equations used to determine the number of lanthanide complexes per particle and the incorporation efficiency]. See DOI: $10.1039 / \mathrm{b} 000000 \mathrm{x} /$

\section{REFERENCES}

75 1. S.W. Bae, W.H. Tan and J.I. Hong, Chem.Commun., 2012, 48, 2270.

2. S. Bonacchi, D. Genovese, R. Juris, M. Montalti, L. Prodi, E. Rampazzo, M. Sgarzi and N. Zaccheroni, Luminescent Chemosensors Based on Silica Nanoparticles, in Luminescence Applied in Sensor Science, 2011, p. 93-138.

80 3. C. Bouzigues, T. Gacoin and A. Alexandrou, ACS Nano, 2011, 5, 8488 .

4. D. Geissler, L.J. Charbonniere, R.F. Ziessel, N.G. Butlin, H.-G. Loehmannsroeben and N. Hildebrandt, Angew. Chem. Int. Ed., 2010, 49, 1396.

85 5. H. Ow, D.R. Larson, M. Srivastava, B.A. Baird, W.W. Webb and U. Wiesner, Nano Letters, 2005, 5, 113-117.

6. V. Holzapfel,A. Musyanovych, K. Landfester, M. R. Lorenz and V. Mailänder, Macromol. Chem. Phys., 2005, 206, 2440.

7. E. Herz, A. Burns, D. Bonner and U. Wiesner, Macromol. Rapid 90 Commun, 2009, 30, 1907.

8. M.Q. Tan, G.L. Wang, X.D. Hai, Z.Q. Ye and J.L. Yuan, J. Mater. Chem., 2004, 14, 2896.

9. A. I. Abderahman, S.C. Thickett, Y. Liang, O. Ornatsky, V. Baranov and M.A. Winnik, Macromolecules, 2011, 44, 4801.

95 10. W. Robert, R.C. Andrew and G.S. David, Angew. Chem. Int. Ed., 2006, 45, 6104.

11. C. Vancaeyzeele, O. Ornatsky, V. Baranov, L. Shen, A. Abdelrahman and M.A. Winnik, J. Am. Chem. Soc., 2007, 129, 3653.

12. H. Goesmann and C. Feldmann, Angew. Chem. Int. Ed., 2010, 49, 1362.

13. J.C.G. Bunzli and C. Piguet, Chem. Soc. Rev., 2005, 34, 1048.

14. S.V. Eliseeva, B. Song, C.D.B. Vandevyver, A.S. Chauvin, J.B. Wacker and J.C.G. Bunzli, New Journal of Chemistry, 2010, 34, 2915.

105 15. J. Samuel, G. Tallec, P. Cherns, W.L. Ling, O. Raccurt, O. Poncelet, D. Imbert and M. Mazzanti, Chem. Commun., 2010, 46, 2647. 
16. J. Desbiens, B. Bergeron, M. Patry and A.M. Ritcey, J. Colloid Interf. Sci., 2012, 376, 12.

17. F. Caruso, in Colloids and Colloid Assemblies,2004, Weinheim: Wiley-VCH.

5 18. D. Urban, and K. Takamura, in Polymer Dispersions and their industrial applications. 2002, Weinheim: Wiley-VCH.

19. M-T. Charreyre, A. Yekta, M.A. Winnik, T. Delair and C. Pichot, Langmuir, 1995, 11, 2423.

20. F. Tronc, M. Li, J.P. Lu, M.A. Winnik, B.L. Kaul and J.C. Graciet, J. Polym. Sci. Part A Polym. Chem., 2003, 41, 766.

21. P. Xu, E. Gullotti, L. Tong, C.B. Highley, D.R. Errabelli, T. Hasan, J.-X. Cheng, D.S. Kohane and Y. Yeo, Molecular Pharmaceutics, 2008, 6, 190.

22. Y.C. Simon, S. Bai, M. K. Sing, H. Dietsch, M. Achermann and C.

15 Weder, Macromol. Rapid Commun., 2012, DOI: 10.1002/marc. 201100708.

23. K. Landfester, Angew. Chem. Int. Ed., 2009, 48, 4488.

24. J.M. Asua, Prog. Polym. Sci., 2002, 27, 1283.

25. K. Landfester, Adv. Mater., 2001,13, 765.

20 26. K. Landfester, N. Bechthold, S. Forster and M. Antonietti, Macromol. Rapid Commun., 1999, 20, 81.

27. L.P. Ramírez, M. Antonietti and K. Landfester, Macromol. Chem. Phys., 2006, 207, 160.

28. K. Ando and H. Kawaguchi, J. Colloid Interf. Sci., 2005, 285, 619.

25 29. E.S. Andreiadis, D. Imbert, J. Pecaut, R. Demadrille and M. Mazzanti, Dalton Transactions, 2012, 41, 1268.

30. N. Wartenberg, M. Mazzanti, D. Imbert, O. Raccurt, Patent FR1256609, submitted 09/07/2012

31. N. Chatterton, Y. Bretonniere, J. Pecaut and M. Mazzanti, Angew. Chem. Int. Ed., 2005, 44, 7595.

32. S. Petoud, S. M. Cohen, J.-C. Bünzli and K. N. Raymond, J. Am. Chem. Soc., 2003, 123, 13324.

33. E. Brunet, O. Juanes, R. Sedano and J.-C. Rodriguez-Ubis, Photochem. Photobiolol. Sci., 2002, 1, 613.

35 34. A. D'Aleo, A. Picot, A. Beeby, J. A. G. Williams, B. Le Guennic, C. Andraud and O. Maury, Inorg. Chem., 2008, 47, 10258.

35. E. G. Moore, J. D. Xu, C. J. Jocher, E. J. Werner and K. N. Raymond, J. Am. Chem. Soc., 2006, 128, 10648.. 